\title{
Investigation of Surface Plasmon Polaritons based Gas sensor: Far and Near field Analysis
}

\section{Sumera Afsheen}

University of Gujrat - Hafiz Hayat Campus: University of Gujrat

\section{Tahir lqbal}

University of Gujrat - Hafiz Hayat Campus: University of Gujrat

Anum Khalid

University of Gujrat - Hafiz Hayat Campus: University of Gujrat

Mohsin ljaz

University of Gujrat - Hafiz Hayat Campus: University of Gujrat

Almas Bashir

University of Gujrat - Hafiz Hayat Campus: University of Gujrat

Muhammad Abrar

University of Gujrat - Hafiz Hayat Campus: University of Gujrat

Muhammad Bilal Tahir

University of Gujrat - Hafiz Hayat Campus: University of Gujrat

\section{Aqsa Tehseen}

University of Gujrat - Hafiz Hayat Campus: University of Gujrat

Maria Zafar ( $D$ maria.zafar76@gmail.com )

University of Gujrat https://orcid.org/0000-0002-3035-4550

\section{Khalid lqbal}

University of Gujrat - Hafiz Hayat Campus: University of Gujrat

Muhammad Rafique

University of Gujrat - Hafiz Hayat Campus: University of Gujrat

\section{Research Article}

Keywords: Refractive index unit (RIU), Biosensing, Attenuated total reflection (ATR), Nanoplasmonics, Finite element analysis (FEA)

Posted Date: March 30th, 2021

DOI: https://doi.org/10.21203/rs.3.rs-350843/v1

License: (9) (1) This work is licensed under a Creative Commons Attribution 4.0 International License. 



\title{
Investigation of Surface Plasmon Polaritons based Gas sensor: Far and Near field Analysis
}

\author{
Sumera Afsheen ${ }^{1},{ }^{+}$Tahir Iqbal ${ }^{2 *},{ }^{+}$Anum Khalid ${ }^{2},{\text { Mohsin } \text { Ijaz }^{2}, \text { Almas Bashir }}^{2}$, Muhammad \\ Abrar $^{3}$, Muhammad Bilal Tahir ${ }^{4}$, Aqsa Tehseen ${ }^{2}$, Maria Zafar ${ }^{2 *}$, Khalid Iqbal ${ }^{5}$, Muhammad \\ Rafique $^{6}$ \\ ${ }^{1}$ Department of Zoology, Faculty of Science, University of Gujrat, Hafiz Hayat Campus, Gujrat, \\ 50700, Pakistan \\ ${ }^{2}$ Department of Physics, Faculty of Science, University of Gujrat, Hafiz Hayat Campus, Gujrat, \\ 50700, Pakistan \\ ${ }^{3}$ Department of Physics, Hazara University, Mansehra, 21300, Pakistan \\ ${ }^{4}$ Department of Physics, Khwaja Fareed University of Engineering and Information Technology, \\ Rahim Yar Khan, Pakistan \\ ${ }^{5}$ Office of Research, Innovation and Commercialization (ORIC), University of Gujrat, Hafiz \\ Hayat Campus, Gujrat, 50700, Pakistan \\ ${ }^{6}$ Department of Physics, University of Sahiwal, Sahiwal, 57000, Pakistan
}

\section{${ }^{+}$Both authors contributed equally.}

*Corresponding authors email: tiqbal02@qub.ac.uk; maria.zafar76@gmail.com

\begin{abstract}
This research work reports the plasmonic-based biosensing using attenuated total reflection (ATR). A photon beam falls on the metallic film through an angle higher than critical angle of glass. SPPs are produced along the metal/analyte interface. An optimum gold ( $\mathrm{Au}$ ) film has been used to sense the refractive index of analyte lying next to it while utilizing the sensitivity power of electric field of the surface plasmon polaritons (SPPs). Such Nanoplasmonic devices have been designed and optimized utilizing finite element analysis (FEA) in licensed version of COMSOL 5.4, RF module. Reflection spectra have been extracted for far-field analysis, whereas shift in the position of SPP dip has been exploited to compute sensitivity of the device. By controlling the film thickness and angle of light incidence, the sensitivity of the device is enhanced. It is worth mentioning that the spectral range was brought in visible region where most of the practical devices work very well. The sensitivity of $18200 \mathrm{~nm} / \mathrm{RIU}$ has been achieved for optimum angle and gold film thickness. Hence such devices have various sensing applications in medical diagnostics.
\end{abstract}




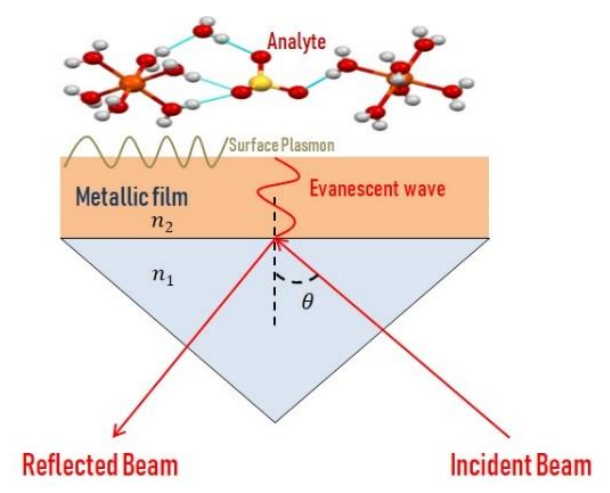

Keywords: Refractive index unit (RIU); Biosensing, Attenuated total reflection (ATR); Nanoplasmonics; Finite element analysis (FEA)

\section{Introduction}

In recent ages, many different techniques have been developed to enhance the sensing quality of gas detection in traces. Sensors play a very attention-grabbing role in several fields such as in environmental monitoring, sensing of explosive gases and biomedical systems. Surface plasmon resonance (SPR) based sensing is an effective technique which has recorded high sensitivities and low background noise. Liedberg [1] and Nylander [2] have made great efforts for the detection of gas with the use of SPR and revealed the details to analyze the behavior of resonant features, such as the spectral shift in transmission and reflection peaks by changing the refractive index of analytes. In biological sciences, biosensor is a tool that is used to detect the chemical changes in analyte [3]. Several researchers are paying attention to biosensors for monitoring of biological interactions. The optical behavior of noble metal nanoparticles can be examined through SPR technique after the development of optical sensors [4]. Optical biosensors employ the SPR phenomenon in which an evanescent wave is used to show the interactions of receptors at the surface of a biosensor and the ligands which are in liquid state on surface. The SPR having evidence as sharp fringes in reflected light is applied to detect the variation in optical properties by altering the refractive index of an analyte. When bimolecular binds to the surface of the biosensor, the density close to the surface changes which in turn gives a change in the SPR angle. The change in this resonance angle offers the base of real-time measurements. The measured variation in the refractive index gives clues about the quantity or density of bound analyte, and its affinity for receptors. The interface between the sensor surface and the biological and chemical entities provides a basis for investigation. 
The most promising technique of attenuated total reflection (ATR) is used to report plasmonicbased biosensing. The sensitivity of biosensors depends upon various parameters such as film thickness of nanostructured devices for maximum sensitivity. The devices have been designed and optimized numerically using the finite element analysis (FEA) method in the RF module of the COMSOL 5.4 licensed version. By controlling the geometry of the unit cell, one can see the significant changes in the field enhancement which is an indicator of the sensitivity of biosensor. The photon beam falls on the Au film through an angle higher than the critical angle of the glass to excite SPPs along with the interface of Au and analyte for sensing purposes. A sharp SPR dip is observed in the reflection spectra and the shift in this dip is indicating the sensitivity of the device.

One of the most developed and commonly used applications of SPPs is biosensing. The rising branch of biosensing has many applications in the experimental analysis [5, 6], medical diagnostics [7], industrial processing control [8, 9], biological warfare weapon control, targeted drug delivery [10], and food quality and safety control [11], etc. Immunoassays based sensing [12] and home pregnancy strips are frequent applications of biosensing.

\section{Background History}

The first in vivo glucose biosensor was designed by Shichiri et al (1980) [13] whose basic design was similar to that of Clark and Layons [14]. Although some conventional biosensors show high sensitivity and specificity such as most frequently used ELISA but these are more complex requiring bulk chemical material, huge and expensive instrumentation. Protein is bio-variable in many chemical and biological processes such as enzyme metabolism and response of immune system. Currently, the nano-biosensors are very helpful for protein revealing as they have multiplexity and ultra-high sensitivity, because they give real-time and label-free measurements. The field-effect device using silicon-nanowire for multiplexed electrical detection of cancer markers has been presented by Lieber's group in 2005[15]. The main problems to be considered include simplicity, speed and cost [16]. Either an antibody or antigen is positioned on solid surface which takes part in a bio-specific interaction to sense and enumerate an analyte of interest. These indirect and direct modes of sensing are branded as heterogeneous and homogeneous immunosensors respectively. 
Direct optical transducers make use of techniques such as SPR, evanescent wave sensing and internal reflectance spectroscopy. Direct optical immunosensors are label free and the sensing wave only penetrates the direct surface of the optical device and does not hinder in the bulk sample $[17,18]$. In these techniques, light entered an optical device from denser medium, falls going on sensing surface and reflected back in the same medium. The reflected light enters in a detector like a photodiode, which offers information with reference to the sensing surface [19]. Indirect immunosensor also finds the exercise of above nominated techniques for sensing. The main advantage of an indirect over direct immunosensors is their improved sensitivity and selectivity.

First time, Kretschmann made the use of SPP for prism coupling in 1968 [20]. In this arrangement, a dip in the measured reflection spectra was observed due to the generation of SP wave which can be used to find the sensitivity response of an SPR sensor. Extraordinary optical transmission (EOT) based sensors are mostly composed of cylindrical holes in metal. Holes in metal are ribbed periodically which boost the sensitivity when the field interacts with the analyte [21].

The sensitivity of biosensor is determined by the ratio of shift in resonant wavelength $\left(\Delta \lambda_{R}\right)$ to variation in refractive index $(\Delta n)$ of the dielectric (Air) and is given by:

$$
S_{R}=\Delta \lambda_{R} / \Delta n
$$

The equation (1) specifies the shift in resonant wavelength with the variation in refractive index of the medium (air) close to metal/analyte interface [5].

For detection of gas, Liedberg [1] and Nylander [2] recommended the utilization of SPR, for example, one dimensional Au grating gas sensor with $599 \mathrm{~nm} / \mathrm{RIU}$ was reported to detect the RI [22]. The sensitivities of surface plasmon based sensors were calculated for $\mathrm{Au}, \mathrm{Ag}$ and $\mathrm{Cu}$ which have values 700, 731 and $722 \mathrm{~nm} \mathrm{RIU}^{-1}$ respectively [23]. Bdour et al obtained sensitivity of 778.6 $\mathrm{nm} / \mathrm{RIU}$ for different sucrose solutions by varying refractive index [24]. SPR based sensor for rectangular and sinusoidal-shaped gratings have sensitivities of 693 and $713 \mathrm{~nm} / \mathrm{RIU}$ [25]. The sensitivity $2170 \mathrm{~nm} / \mathrm{RIU}$ of SPR based fibre optic sensor working on Kretschmann configuration was reported [26]. The refractive index sensor achieved the sensitivity $2600 \mathrm{~nm} \mathrm{RIU}{ }^{-1}$ for blood glucose detection [27]. For both gas and liquid detection, Lu et al measured the waveguide coupled SPR sensor showing $9000 \mathrm{~nm} / \mathrm{RIU}$ [28]. SnSe-graphene hybrid photonic SPR sensor achieved the 94.29 deg. RIU $^{-1}$ [29]. Qi and Zhang et al had evaluated a bilayer graphene refractive index sensor with sensitivity $14110 \mathrm{~nm} / \mathrm{RIU}[30,31]$. 


\section{Numerical Modelling}

A 2D model is designed in COMSOL 5.4, RF module to get reflection spectra for this research work in which finite element analysis (FEA) is used. A p-polarized light of wavelength ' $\lambda$ ' range $400-1200 \mathrm{~nm}$ is incident on a thin film of Au placed on a glass substrate $(\mathrm{n}=1.5)$ with optimized incidence angle $\left(43^{\circ}\right)$. The structural parameters, such as film thickness ' $t$ ' varying from $50 \mathrm{~nm}$ to $200 \mathrm{~nm}$ is selected. The refractive index ' $\mathrm{n}$ ' of air analyte is varying from 1.00-1.008. The schematic of this device with all boundary conditions is shown in Fig. 1. The resolution for fine regions is $1 \mathrm{~nm}$ with element size $20 \mathrm{~nm}$. The additional numerical factors involve $1.3 \mathrm{~nm}$ growth rate of elements, 0.3 mesh factor, $1 \mathrm{~nm}$ element size of scaling factor. The periodic boundary conditions (PBCs) represent the peripheral borders of the model. The application of PBCs can be observed for the huge systems which exhibit translational symmetry on very small length scale and is calculated by a single unit cell. Every bit of the facts regarding the fields is obtained by cycling the phase of the solution within infinite structure of a unit cell.

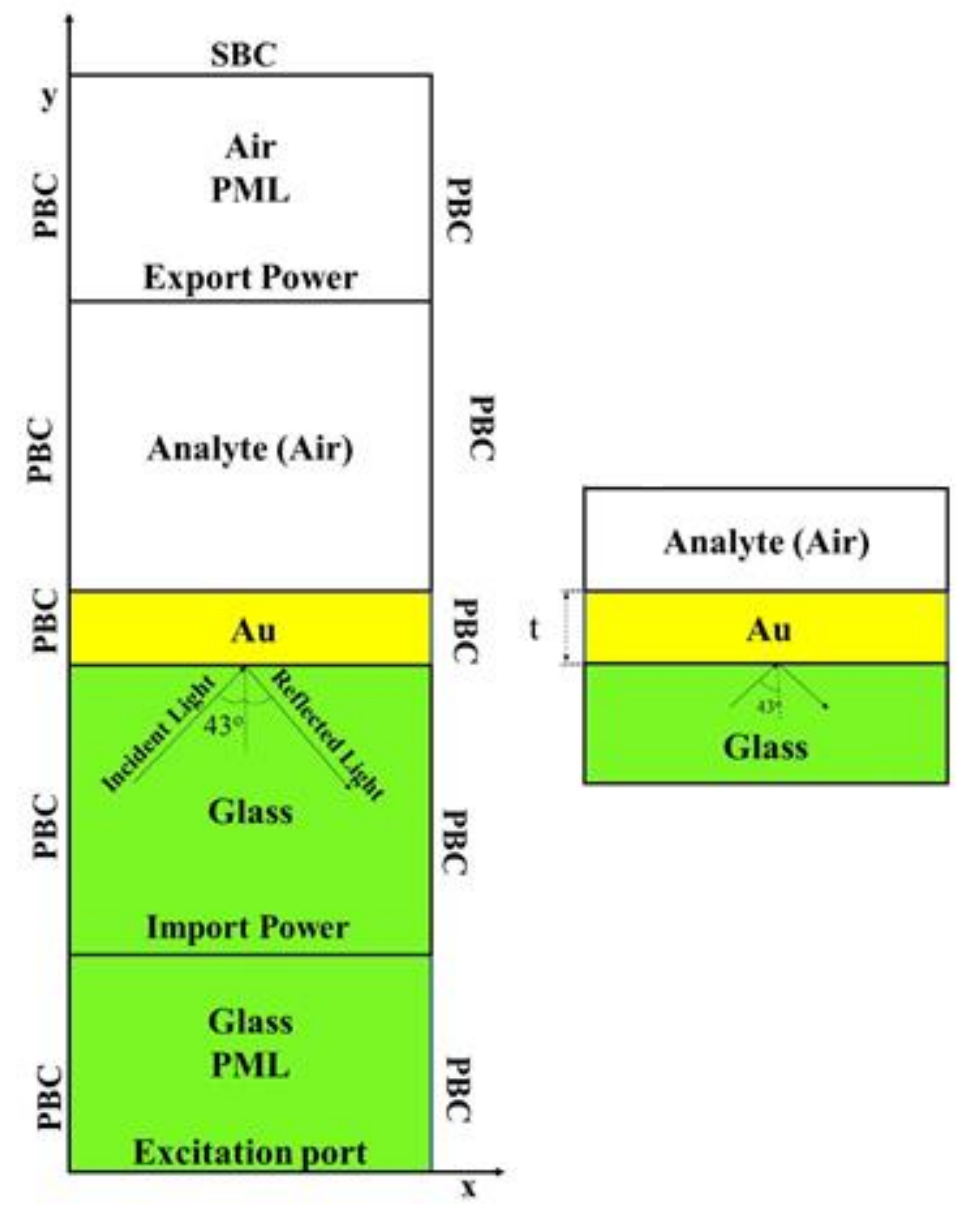

Fig. 1: A 2D model designed in COMSOL Multiphysics to get reflection spectra for this research work where right side a single unit cell with optimized incidence angle is $43^{\circ}$, and film thickness is a varying parameter (50- $200 \mathrm{~nm}$ ). 
The effect of phase shift at each boundary produced by tangential components is considered using Floquent periodicity in combination with PBCs. These components of EM fields of a model are ensured by continuity boundary conditions (CBCs) which represent the interior boundaries of that model [32-38]. The incident wave vector, designation boundary to source distance and material properties have significant effect on phase shift. The significance of Floquent periodicity is much more for the incoming wave striking at some angle rather than falling normally because for normal incidence PBCs along with continuity periodicity is sufficient [39]. When the reflection is required to be compact, the boundary conditions applied are scattering boundary conditions. If incident fields are not properly absorbed due to insufficient scattering boundaries and large spread $k$ vectors then perfectly matched layers (PMLs) are applied for bounding the interior calculation space at the final continuity boundaries which expand the computation domain $\sim \lambda / 2$. [40]. The EM wave with sinusoidally-varying amplitude is reported experimentally for the excitation of 1D grating which corresponds to Harmonic propagation mode. The expression for the wave having instantaneously changing amplitude is of form;

$$
\Psi(x)=e^{-i k_{x} x}
$$

where $k_{x}=n k_{o} \sin \theta_{i}, k_{o}=\frac{2 \pi}{\lambda_{o}}$ and $\lambda_{o}$ is the incident wavelength [41], and $\theta_{i}$ is the incident angle taken normal to the surface. To proceed for modelling of this research work, firstly the model is authenticated by various theoretical results of literature which are already proven true.

\section{Results and Discussion}

To comprehend the excitation and working of surface plasmon (SPs) on Au/analyte interface, the attenuated total reflection technique is used. The SPs are in fact electromagnetic excitations that yield up at the interface of metal/dielectric. When momentum of SPs matches with the momentum of incoming photon, SPs are excited to give a very interesting phenomena called as surface plasmon resonance (SPR). This phenomenon is very useful for biosensing because it gives accurate measurements of alterations in refractive index or sensing of analyte attached to the metal film. The thickness of gold film is optimized to use as biosensor by measuring the sensitivity which is widely used by many researchers for its high chemical strength and elevated sensitivity [42]. The $\mathrm{k}$-vector matching technique to give SPR used in this work is attenuated total reflection or Kretschmann configuration.

\section{Kretschmann Configuration}


When wave-vector component of p-polarized light along the Au-film equivalents with the wavevector of SPs at the Au surface, SPPs are produced. Due to this matching of wave vectors, photon of p-polarized light is coupled with SPs at wavelength. In order to get dispersion relation of SPPs, Maxwell's equations are used for incident light. The wave-vector associated with the light and surface plasmon polaritons are given by equation (3), which is well known propagation relation of SPPs [41].

$$
k_{S P P}=\frac{2 \pi}{\lambda_{o}} \sqrt{\frac{\varepsilon_{m} \varepsilon_{d}}{\varepsilon_{m}+\varepsilon_{d}}}
$$

where $\lambda_{o}$ is the incident wavelength, $\varepsilon_{d}$ and $\varepsilon_{m}$ are complex dielectric constants of dielectric and metals.

In this configuration, the matching of wave-vectors is attained by increasing the wave vector of light when it passes through glass prism as given in Kretschmann-Raether equation (4) [41].

$$
k_{x}=\frac{2 \pi}{\lambda_{o}} n_{p} \sin \theta_{i}
$$

where $n_{p}$ is RI of prism and $\theta_{i}$ is angle of incidence.

The SPP resonance is very exciting optical response of the metals. In this research work, the film thickness of $\mathrm{Au}$ is optimized at glass substrate for getting the maximum sensitivity of biosensor. This is done by field enhancement at the surface of the Au film for different film thickness from 50 to $200 \mathrm{~nm}$, which is described in the section near field analysis. A sharp dip in the reflectance spectra indicates the condition of SPP excitation[43-45]. In principle there are four parameters that can give us clues for sensitivity measurement in SPR sensing which are wavelength interrogation (variation in the resonance wavelength for fixed angle of incidence), intensity interrogation (variation in the reflectance for fixed angle of incidence), angular interrogation (variation in resonance angle) and phase interrogation. The first method i.e., wavelength interrogation is selected to investigate the sensitivity measurement of sensor device.

\section{Far Field Analysis}

The reflection spectra are obtained through nano-structured Au film of varying thickness of 50, 100, 150 and $200 \mathrm{~nm}$, a fixed angle of incidence $\left(43^{\circ}\right)$. The sensing of analyte can be noticed simply in the Fig. 2 (a, b, c, and d), the shifting of reflection resonance dips lies in the wavelength range $750-950 \mathrm{~nm}$, while RI of adjacent media is varied from 1.00 to 1.008 . For very small change in RI of analyte, a corresponding change occurs in the dielectric constant of analyte $\left(n^{2}=\varepsilon_{d}\right)$ 
nearby metal film. Hence, there is shift in resonance wavelength $\left(\lambda_{\text {res }}=\frac{2 \pi}{k_{S P P}}\right)$ indicated in equation (3) which is very useful for sensing applications.
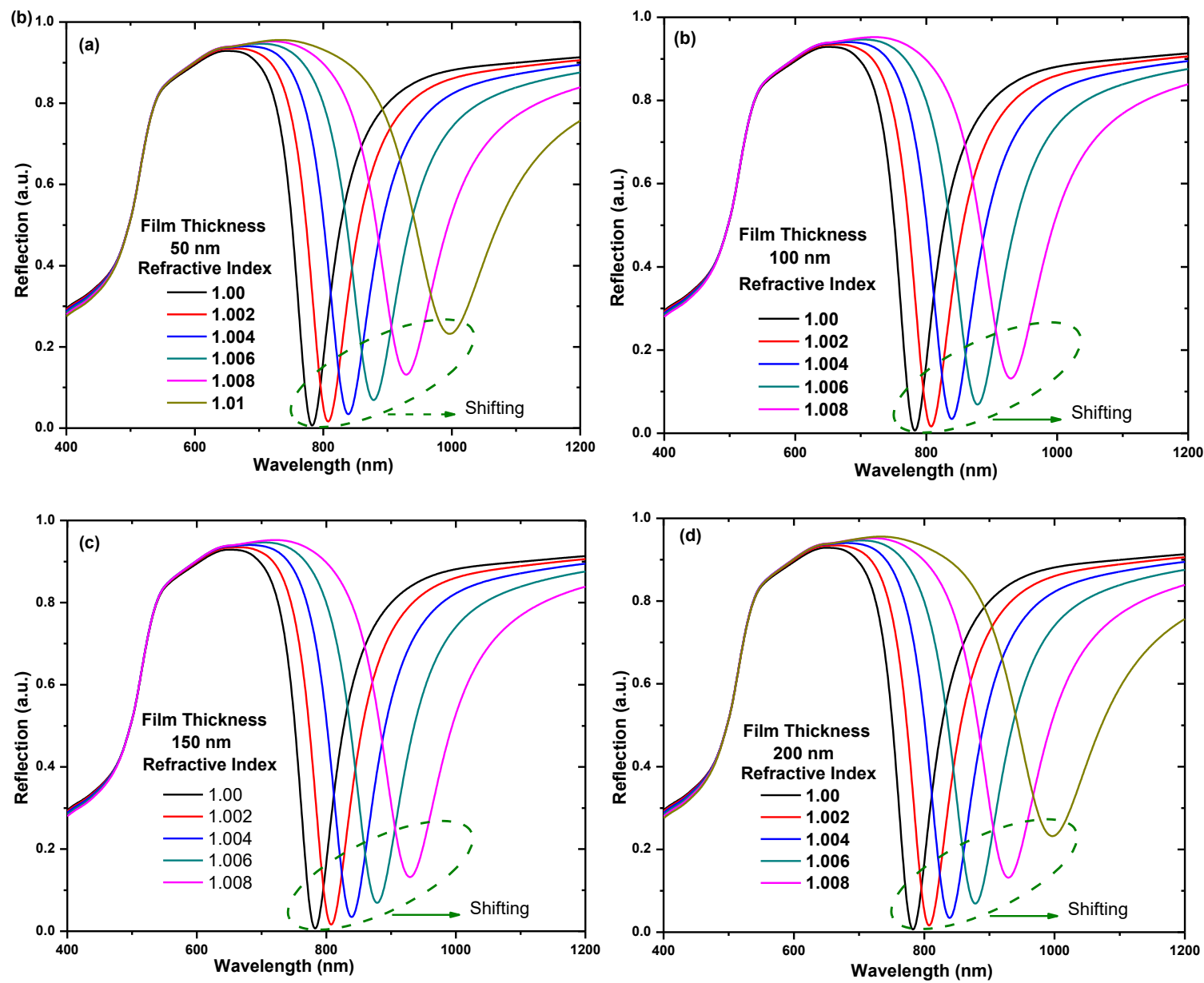

Fig. 2: Reflection spectra of p-polarized light through gold film in the wavelength range (400-1200 nm) at the angle $43^{\circ}$ beyond the critical angle for film thickness (a) $50 \mathrm{~nm}$ (b) $100 \mathrm{~nm}$ (c) $150 \mathrm{~nm}$ (d) $200 \mathrm{~nm}$. 
The shifting in the characteristic dips is clearly seen which can be used to calculate the sensing by using equation 1. A direct relationship exists between the shifting of SPP resonance to higher wavelength and a very slight variation in RI of analyte. The slope between SPP resonance wavelength and RI is calculated for the film thickness of $50 \mathrm{~nm}$ (Fig. 3a) and $200 \mathrm{~nm}$ (Fig. 3b), which is a straight line. Similar straight line is also observed for film thickness of $100 \mathrm{~nm}$ and 150
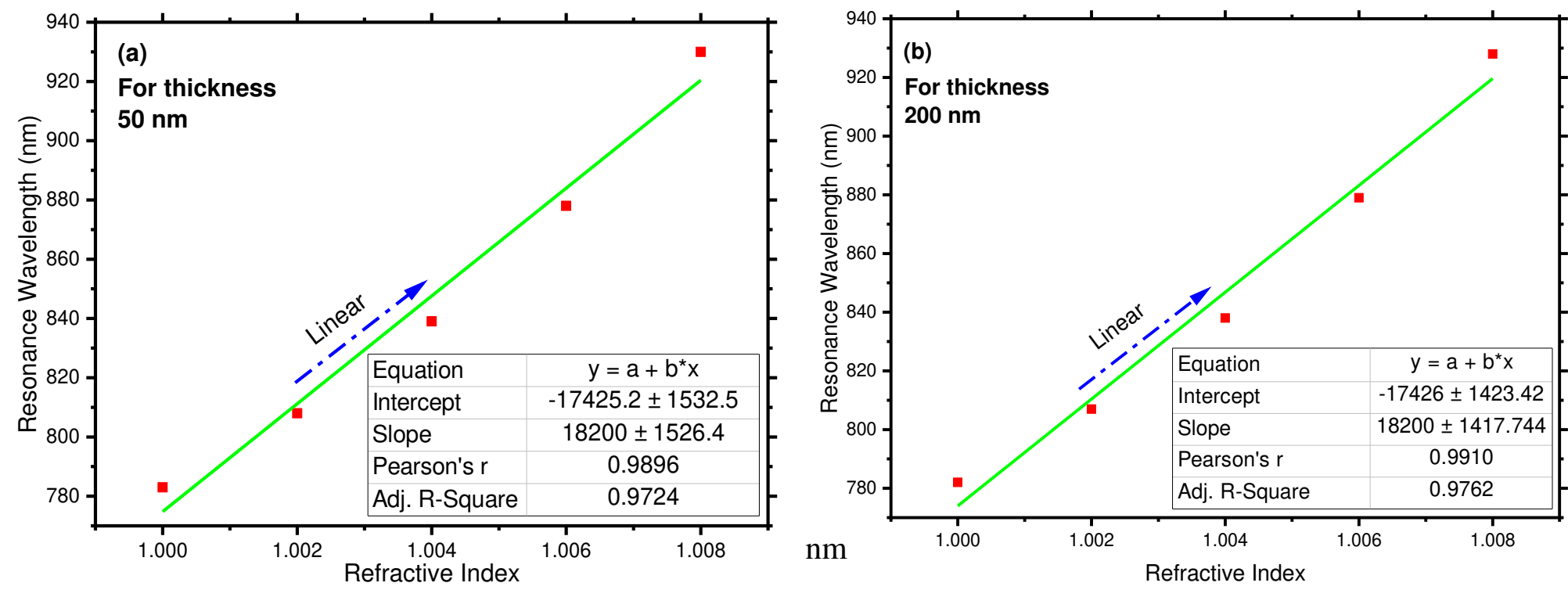

(not shown here). This direct relation shown in Fig. 3 in the form of straight line becomes the base of measuring $18200 \mathrm{~nm} / \mathrm{RIU}$ sensitivity of such device.

Fig. 3: SPP resonance wavelength versus refractive index (RI) of analyte for film thickness of (a) $50 \mathrm{~nm}$ and (b) 200 nm.

\section{Near Field Analysis}

The 2D plots of magnitude of y-component of electric field $\left(E_{y}\right)$ and z-component of magnetic field $\left(B_{z}\right)$ at the film thickness $50 \mathrm{~nm}$ is shown in Fig. 4. It is observed that the variation in the refractive index of analyte is as 1.00, 1.002, 1.004, 1.006, 1.008 and 1.01 and the respective resonance wavelengths are 783, 808, 839, 878, 930 and $997 \mathrm{~nm}$. These plots show that fields are more concentrated at film thickness of $50 \mathrm{~nm}$. 
(a) $n=1, \lambda=783$

(b) $n=1, \lambda=783$

(c) $n=1.002, \lambda=808$

(d) $n=1.002, \lambda=808$ 


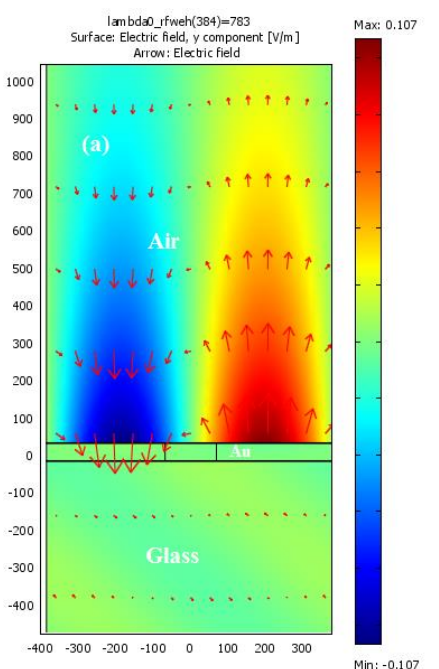

(e) $n=1.004, \lambda=839$

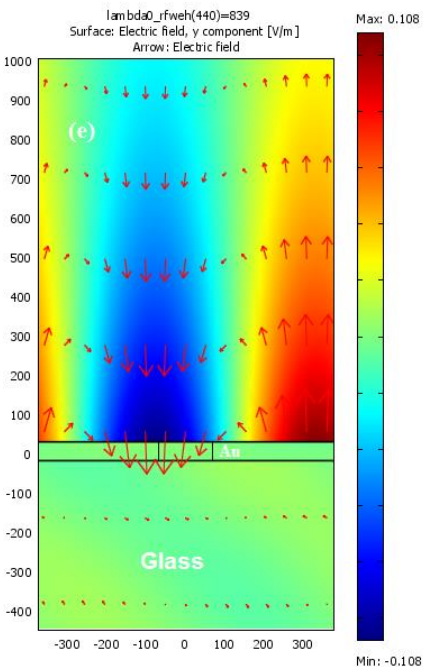

(i) $\mathrm{n}=1.008, \lambda=930$

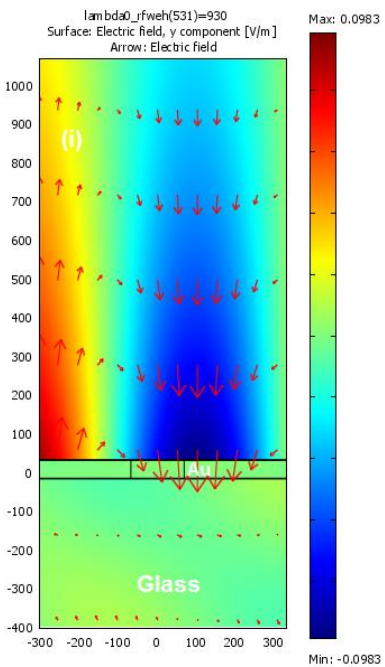

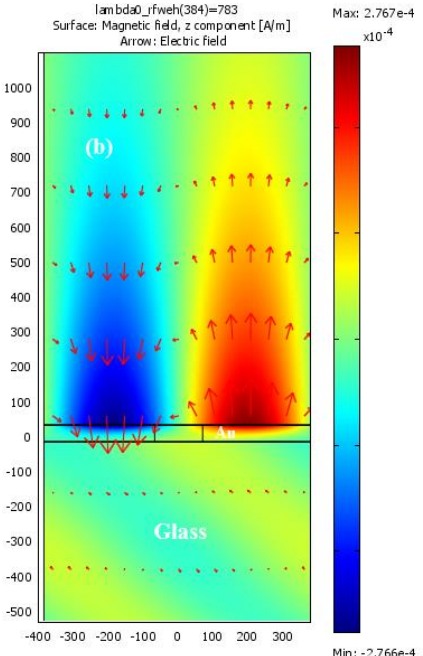

(f) $n=1.004, \lambda=839$

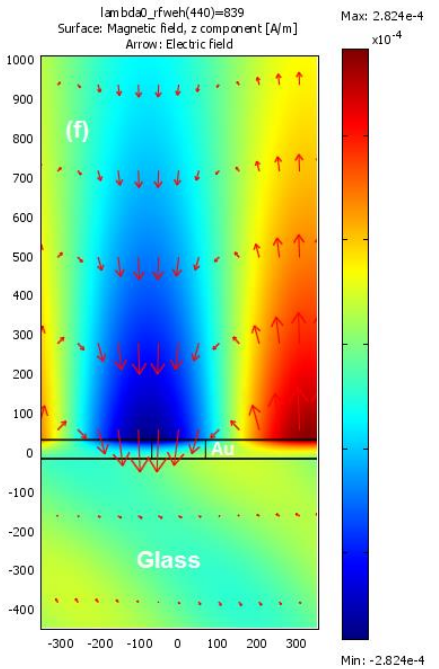

(j) $n=1.008, \lambda=930$

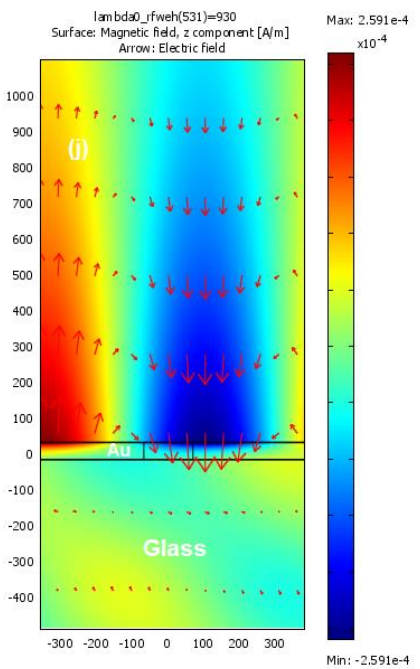

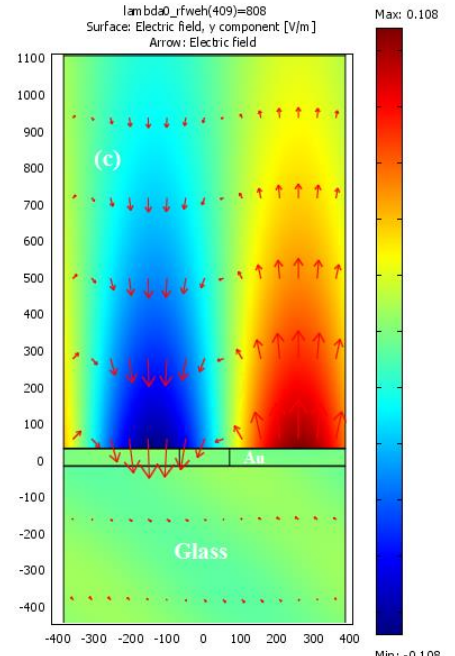

(g) $\mathrm{n}=1.006, \lambda=878$

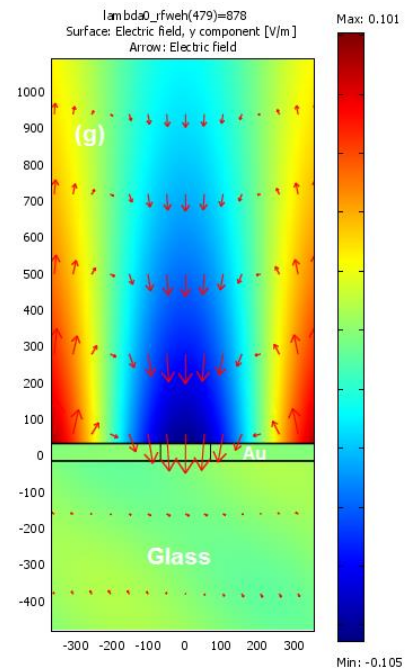

(k) $\mathrm{n}=1.01, \lambda=997$

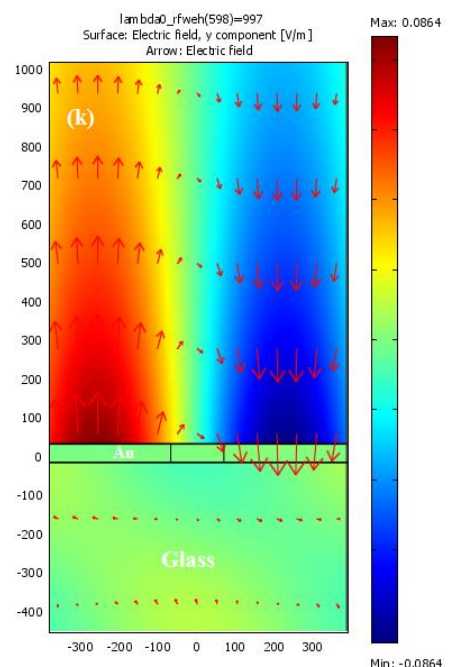

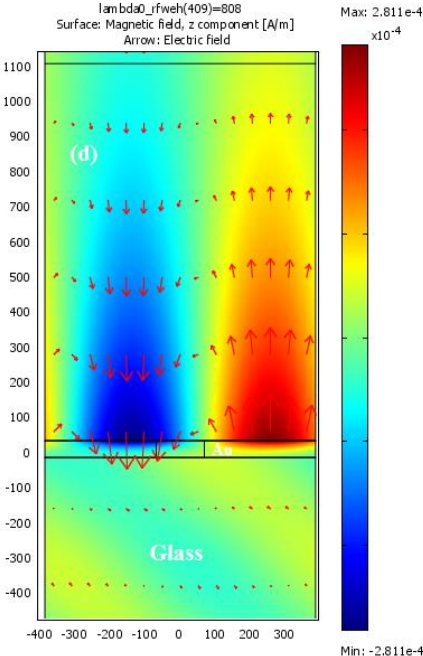

(h) $\mathrm{n}=1.006, \lambda=878$

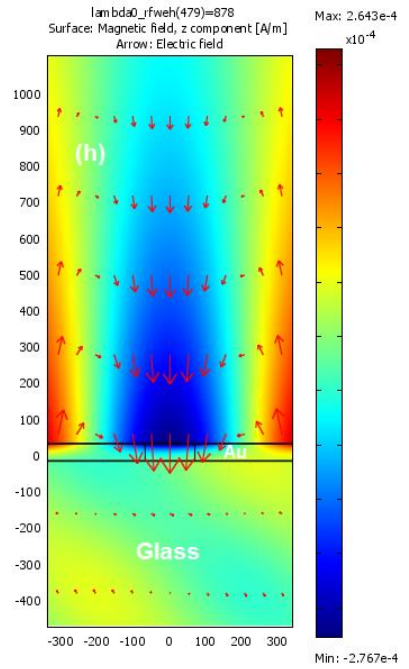

(I) $\mathrm{n}=1.01, \lambda=997$

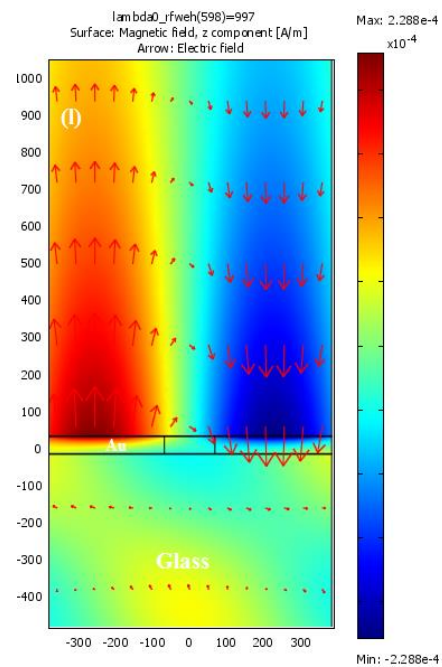


Fig. 4: y-component of electric field and z-component of magnetic field at incidence $43^{\circ}$ with film thickness $50 \mathrm{~nm}$. The y-components of electric field $\left(E_{y}\right)$ are shown in Fig. 4 (a, c, e, g, i, k) and z-components of magnetic field are shown in Fig. 4 (b, d, f, h, j, l).

To determine the field enhancement, $E_{y}$ is plotted against wavelength spectrum range for different film thickness as shown in Fig. 5 (a). The electric field enhancement is maximum for $50 \mathrm{~nm}$ gold film and then there is decrease in the field penetration up to $150 \mathrm{~nm}$. At $50 \mathrm{~nm}$ gold film thickness, the penetration of the field is maximum indicating strong evidence of SPPs. So, optimization of $50 \mathrm{~nm}$ gold film is achieved as shown in Fig. 5. Below $50 \mathrm{~nm}$ film thickness, it approaches to skin depth of the metal (gold) and SPPs are not efficient enough. There is an increasing trend of field enhancement after $150 \mathrm{~nm}$ but excessive use of material in higher film thickness is not suitable, so this is not an area of interest indicating by an arrow B shown in Fig. 5 (b).
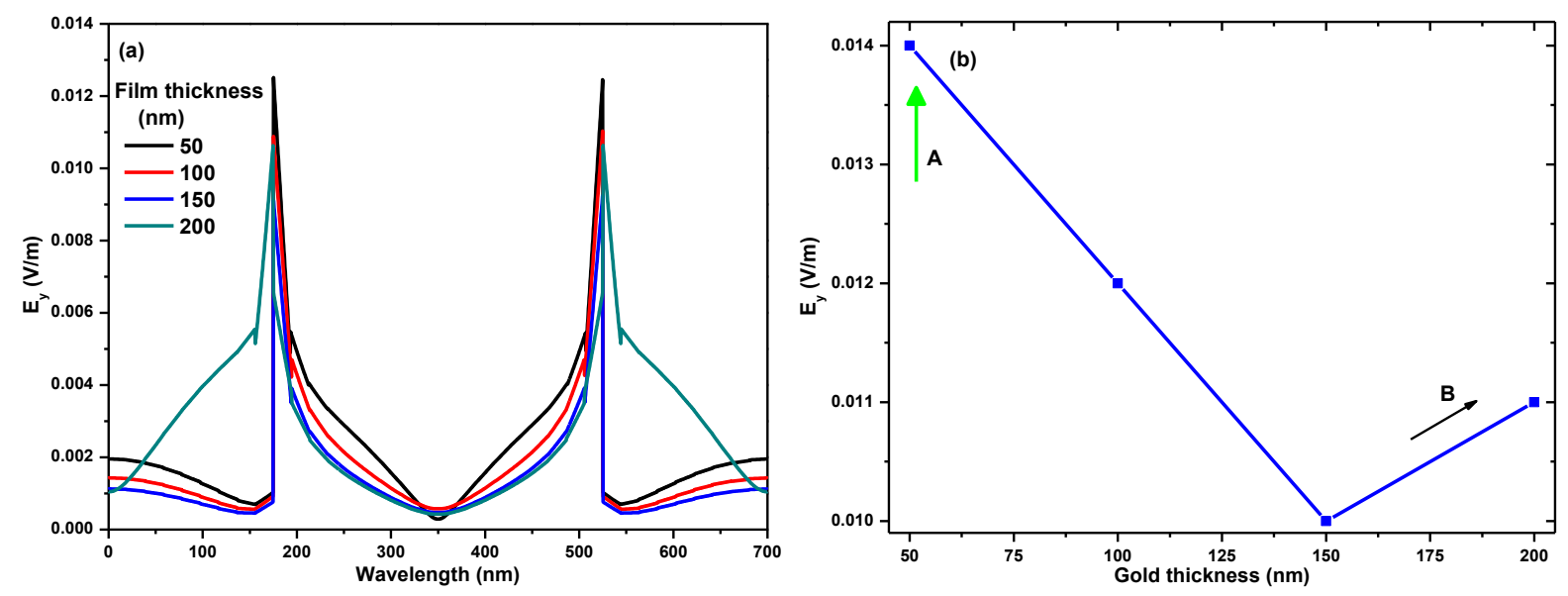

Fig. 5: Cross sectional view of the electric field at different gold thicknesses. (a) y-component of electric field versus wavelength, (b) y-component of electric field versus gold thickness.

\section{Conclusion}

This study has analyzed the sensitivity of existing SPR biosensors using ATR technique for most used detection method wavelength interrogation. To measure very slight variation in the refractive index of adjacent media, the optimization of gold film thickness $50 \mathrm{~nm}$ is achieved at an angle beyond the critical angle i.e. $43^{\circ}$. The sensitivity $18200 \mathrm{~nm} / \mathrm{RIU}$ is obtained which is much higher than previously reported ones. The excitations of SPPs have been achieved with transverse magnetic (TM) wave only, while no SPPs by transverse electric (TE) wave. The penetration of fields associated with the SPPs is very sensitive to detect the change in the refractive index of adjacent media and determines potential application in the field of bio-sensing. It is concluded that 
sensitivity is related to the film thickness and gives maximum field enhancement for $50 \mathrm{~nm}$ film thickness.

\section{Conflict of Interest}

On behalf of all authors, the corresponding author states that there is no conflict of interest.

\section{References}

[1] B. Liedberg, I. Lundström, E. Stenberg, Principles of biosensing with an extended coupling matrix and surface plasmon resonance, Sensors and Actuators B: Chemical, 11 (1993) 63-72.

[2] B. Liedberg, C. Nylander, I. Lundström, Biosensing with surface plasmon resonance-how it all started, Biosensors and Bioelectronics, 10 (1995) i-ix.

[3] I. Karube, Micro-organism based sensors, inBiosensors: Fundamentals and Application (Turner, APF, Karube, I., and Wilson, GS, eds.), Oxford University Press, Oxford, 1987.

[4] L.A. Terry, S.F. White, L.J. Tigwell, The application of biosensors to fresh produce and the wider food industry, Journal of agricultural and food chemistry, 53 (2005) 1309-1316.

[5] M. Hong, L. Shi, H. Li, Y. Du, Z. Wang, Y. Weng, D. Li, Resonance in a sub-wavelength metal-dielectric free-standing grating utilized for gas sensors, Optics Communications, 285 (2012) 5480-5485.

[6] P. D'Orazio, Biosensors in clinical chemistry, Clinica Chimica Acta, 334 (2003) 41-69.

[7] S. Rodriguez-Mozaz, M.J.L. de Alda, D. Barceló, Biosensors as useful tools for environmental analysis and monitoring, Analytical and bioanalytical chemistry, 386 (2006) 1025-1041.

[8] D. Sahoo, A. Mandal, T. Mitra, K. Chakraborty, M. Bardhan, A.K. Dasgupta, Nanosensing of pesticides by zinc oxide quantum dot: an optical and electrochemical approach for the detection of pesticides in water, Journal of agricultural and food chemistry, 66 (2018) 414-423.

[9] A.J. Baeumner, Biosensors for environmental pollutants and food contaminants, Analytical and bioanalytical chemistry, 377 (2003) 434-445.

[10] D. Yu, B. Blankert, J.C. Viré, J.M. Kauffmann, Biosensors in drug discovery and drug analysis, Analytical letters, 38 (2005) 1687-1701.

[11] J.J. Gooding, Biosensor technology for detecting biological warfare agents: Recent progress and future trends, Analytica Chimica Acta, 559 (2006) 137-151.

[12] J. Sambles, G. Bradbery, F. Yang, Optical excitation of surface plasmons: an introduction, Contemporary physics, 32 (1991) 173-183.

[13] M. Shichiri, Y. Yamasaki, R. Kawamori, N. Hakui, H. Abe, Wearable artificial endocrine pancreas with needle-type glucose sensor, The Lancet, 320 (1982) 1129-1131. 
[14] L.C. Clark Jr, C. Lyons, Electrode systems for continuous monitoring in cardiovascular surgery, Annals of the New York Academy of sciences, 102 (1962) 29-45.

[15] G. Zheng, F. Patolsky, Y. Cui, W.U. Wang, C.M. Lieber, Multiplexed electrical detection of cancer markers with nanowire sensor arrays, Nature biotechnology, 23 (2005) 1294.

[16] J. Pearson, A. Gill, P. Vadgama, Analytical aspects of biosensors, Annals of clinical biochemistry, 37 (2000) 119-145.

[17] P.B. Luppa, L.J. Sokoll, D.W. Chan, Immunosensors-principles and applications to clinical chemistry, Clinica Chimica Acta, 314 (2001) 1-26.

[18] C.L. Morgan, D.J. Newman, C. Price, Immunosensors: technology and opportunities in laboratory medicine, Clinical Chemistry, 42 (1996) 193-209.

[19] D. Purvis, D. Pollard-Knight, P. Lowe, Biosensors based on evanescent waves, CHEMICAL ANALYSISNEW YORK-INTERSCIENCE THEN JOHN WILEY-, 1 (1998) 165-226.

[20] E. Kretschmann, H.J.Z.f.N.A. Raether, Radiative decay of non radiative surface plasmons excited by light, 23 (1968) 2135-2136.

[21] A. Li, X. Wang, L. Guo, S.J.T.J.o.P.C.C. Li, Tunable Subradiant Mode in Free-Standing Metallic Nanohole Arrays for High-Performance Plasmofluidic Sensing, 123 (2019) 25394-25401.

[22] S. Afsheen, T. Iqbal, M. Aftab, A. Bashir, A. Tehseen, M.Y. Khan, M.J.M.R.E. Ijaz, Modeling of 1D Au plasmonic grating as efficient gas sensor, 6 (2019) 126203.

[23] S. Afsheen, T. Iqbal, S. Akram, A. Bashir, A. Tehseen, M. Rafique, M. Shakil, M.Y. Khan, M.J.O. ljaz, Q. Electronics, Surface plasmon based 1D-grating device for efficient sensing using noble metals, 52 (2020) 64.

[24] Y. Bdour, C. Escobedo, R.G.J.O.e. Sabat, Wavelength-selective plasmonic sensor based on chirpedpitch crossed surface relief gratings, 27 (2019) 8429-8439.

[25] T. Iqbal, S. Noureen, S. Afsheen, M.Y. Khan, M.J.O.M. Ijaz, Rectangular and sinusoidal Au-Grating as plasmonic sensor: A comparative study, 99 (2020) 109530.

[26] V. Kapoor, N.K.J.M. Sharma, O.T. Letters, Surface plasmon resonance-based fiber optic sensor prepared from bilayers of indium tin oxide-indium oxide, DOI (2020).

[27] S.K. Chamoli, S. Singh, C.J.I.S.J. Guo, Design of extremely sensitive refractive index sensors in infrared for blood glucose detection, DOI (2020).

[28] D.-Y. Lu, W. Li, H. Zhou, X. Cao, Y. Zhu, K.-J. Wang, H.-J. Luo, J.-B. Li, X.-M. Zhang, M.-D.J.P. He, Waveguide-Coupled Surface Plasmon Resonance Sensor for Both Liquid and Gas Detections, DOI (2020) $1-9$. 
[29] M.S. Rahman, K. Rikta, L.F. Abdulrazak, M.J.P. Anower, Nanostructures-Fundamentals, Applications, Enhanced performance of SnSe-Graphene hybrid photonic surface plasmon refractive sensor for biosensing applications, 39 (2020) 100779.

[30] Y. Qi, Y. Zhang, C. Liu, T. Zhang, B. Zhang, L. Wang, X. Deng, Y. Bai, X.J.R.i.P. Wang, A tunable terahertz metamaterial absorber composed of elliptical ring graphene arrays with refractive index sensing application, 16 (2020) 103012.

[31] Y. Qi, Y. Zhang, C. Liu, T. Zhang, B. Zhang, L. Wang, X. Deng, X. Wang, Y.J.N. Yu, A Tunable Terahertz Metamaterial Absorber Composed of Hourglass-Shaped Graphene Arrays, 10 (2020) 533.

[32] T. Iqbal, Propagation length of surface plasmon polaritons excited by a 1D plasmonic grating, Current Applied Physics, 15 (2015) 1445-1452.

[33] T. Iqbal, S. Afsheen, Plasmonic band gap: role of the slit width in 1D metallic grating on higher refractive index substrate, Plasmonics, 11 (2016) 885-893.

[34] T. Iqbal, S. Afsheen, Coupling efficiency of surface plasmon polaritons for 1D plasmonic gratings: role of under-and over-milling, Plasmonics, 11 (2016) 1247-1256.

[35] T. Iqbal, S. Afsheen, Extraordinary optical transmission: Role of the slit width in 1D metallic grating on higher refractive index substrate, Current Applied Physics, 16 (2016) 453-458.

[36] M. Javaid, T. Iqbal, Plasmonic bandgap in 1D metallic nanostructured devices, Plasmonics, 11 (2016) 167-173.

[37] T. Iqbal, Coupling efficiency of surface plasmon polaritons: far-and near-field analyses, Plasmonics, 12 (2017) 215-221.

[38] T. Iqbal, M. Ijaz, M. Javaid, M. Rafique, K.N. Riaz, M.B. Tahir, G. Nabi, M. Abrar, S. Afsheen, An Optimal Au Grating Structure for Light Absorption in Amorphous Silicon Thin Film Solar Cell, Plasmonics, DOI (2018) 1-8.

[39] T. Iqbal, Nanoplasmonic grating coupler for transducer applications, Queen's University Belfast, 2013.

[40] D. O'Connor, Modelling of nano-optic light delivery mechanisms for use in high density data storage, Queen's University Belfast, 2010.

[41] H. Raether, Surface Plasmons on Smooth and Rough Surfaces and on Gratings, 1988.

[42] G. Strang, G.J. Fix, An analysis of the finite element method, Prentice-hall Englewood Cliffs, NJ1973.

[43] S. Vempati, T. Iqbal, S. Afsheen, Non-universal behavior of leaky surface waves in a one dimensional asymmetric plasmonic grating, Journal of Applied Physics, 118 (2015) 043103.

[44] T. Iqbal, S. Afsheen, One dimensional plasmonic grating: high sensitive biosensor, Plasmonics, 12 (2017) 19-25. 
[45] M. Munir, M.I. Khan, T. Iqbal, M. Abrar, M.B. Tahir, J. ur Rehman, M. ljaz, G. Nabi, Efficient biosensing through 1D silver nanostructured devices using plasmonic effect, Nanotechnology, DOI (2018). 
Figures

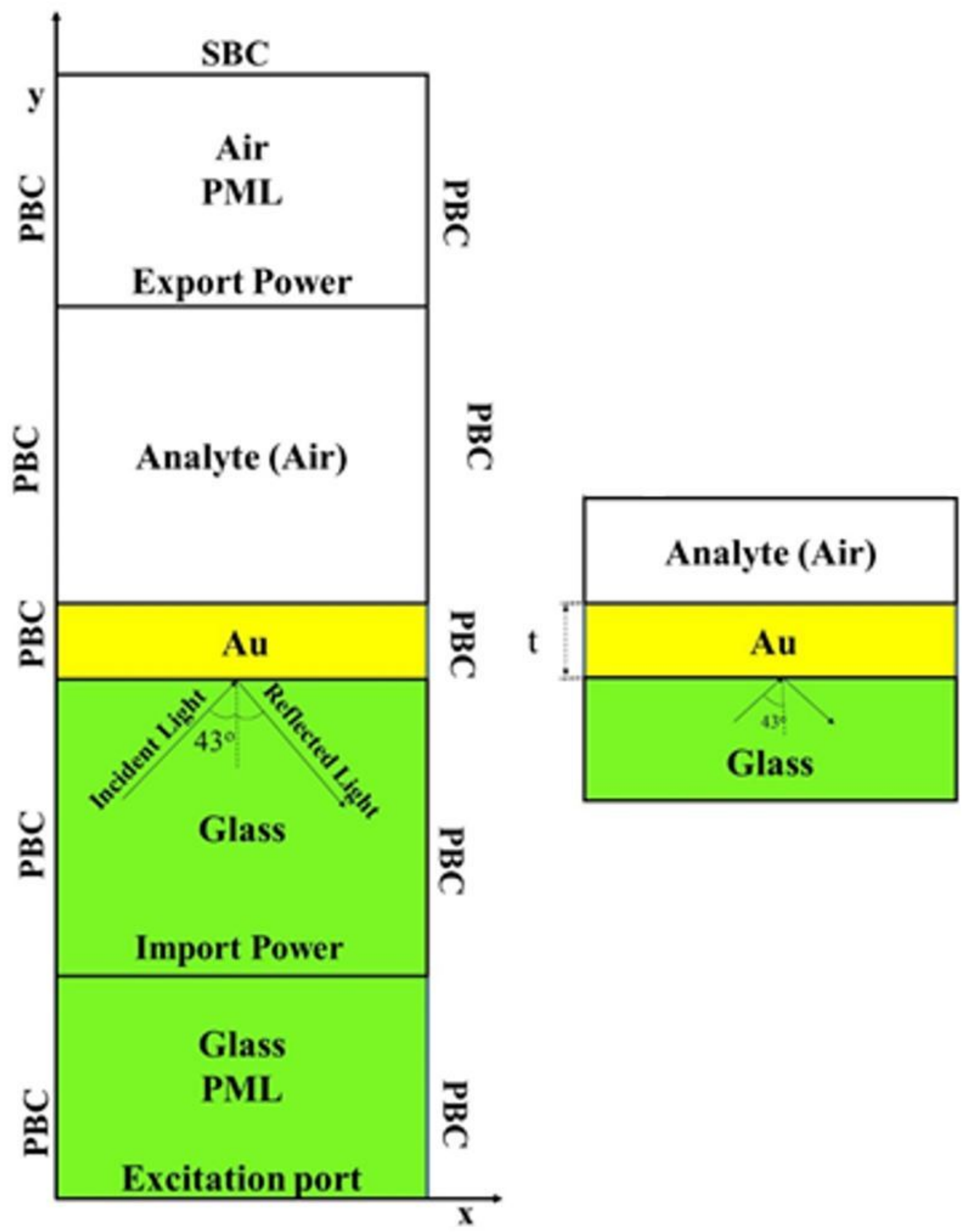

Figure 1

A 2D model designed in COMSOL Multiphysics to get reflection spectra for this research work where right side a single unit cell with optimized incidence angle is 430 , and film thickness is a varying parameter (50- $200 \mathrm{~nm}$ ). 

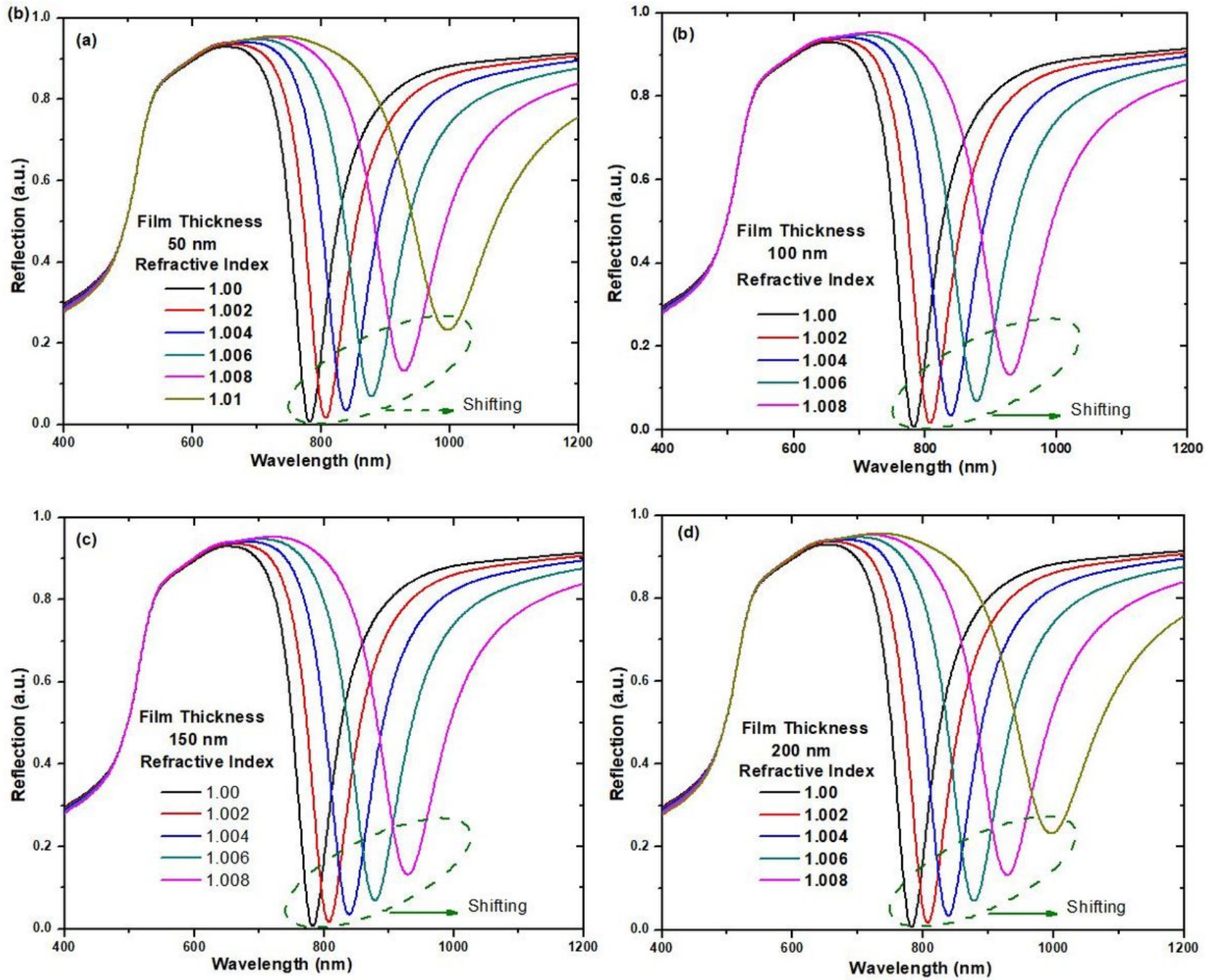

Figure 2

Reflection spectra of p-polarized light through gold film in the wavelength range (400-1200 nm) at the angle 430 beyond the critical angle for film thickness (a) $50 \mathrm{~nm}$ (b) $100 \mathrm{~nm}$ (c) $150 \mathrm{~nm}$ (d) $200 \mathrm{~nm}$. 

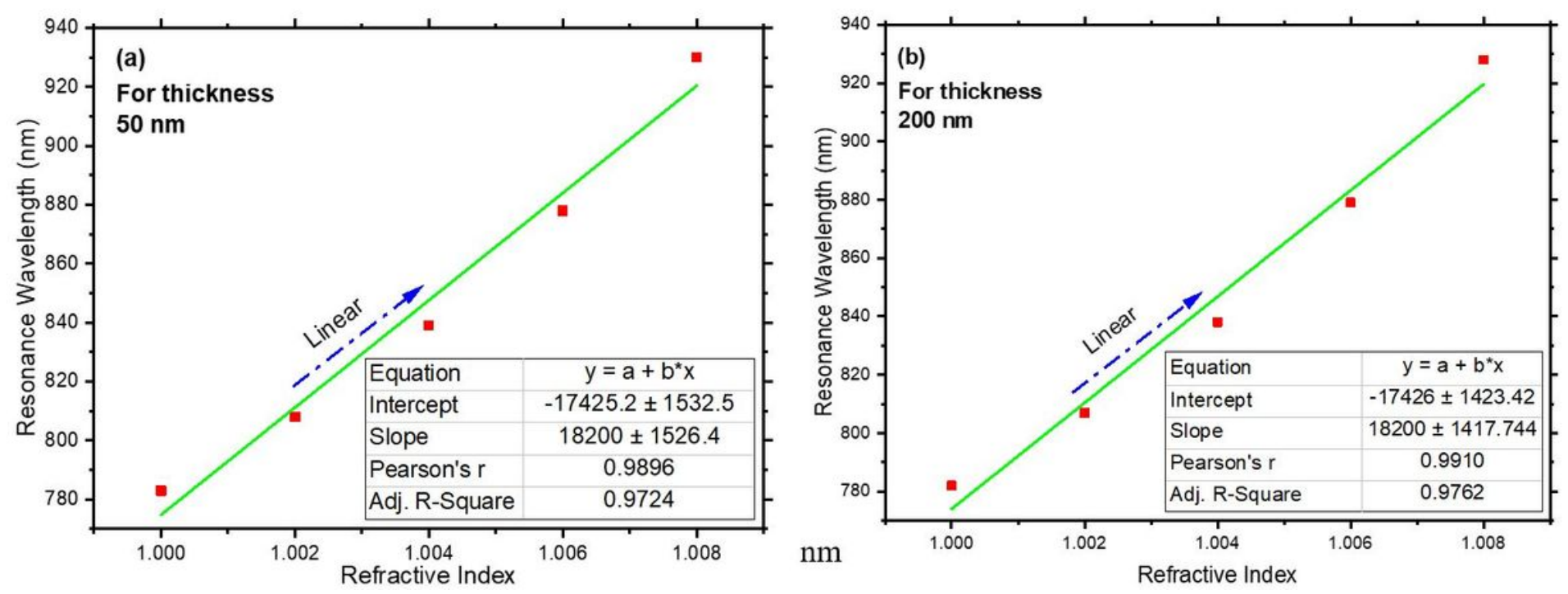

Figure 3

SPP resonance wavelength versus refractive index (RI) of analyte for film thickness of (a) $50 \mathrm{~nm}$ and (b) $200 \mathrm{~nm}$. 
(a) $n=1, \lambda=783$

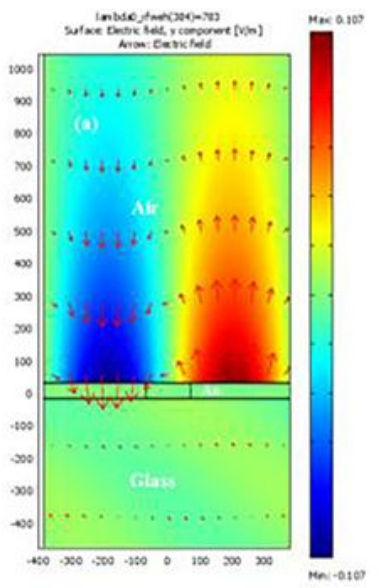

(e) $n=1.004, \lambda=839$

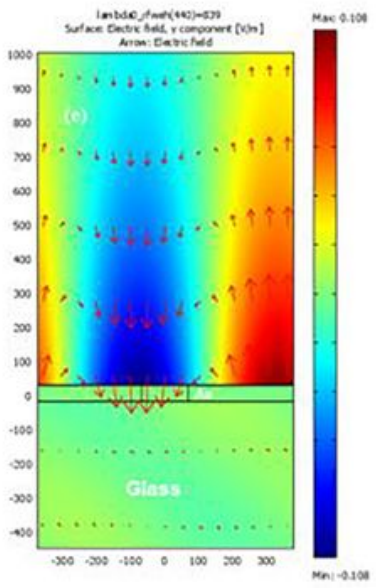

(i) $\mathrm{n}=1.008, \lambda=930$

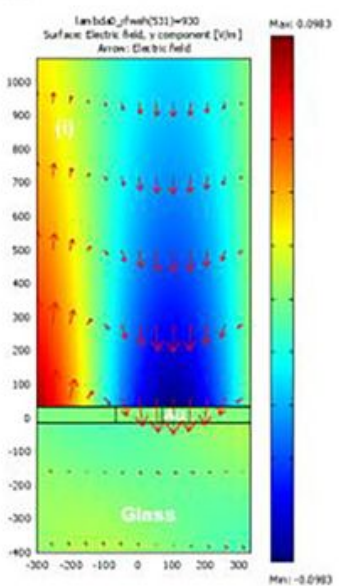

(b) $n=1, \lambda=783$

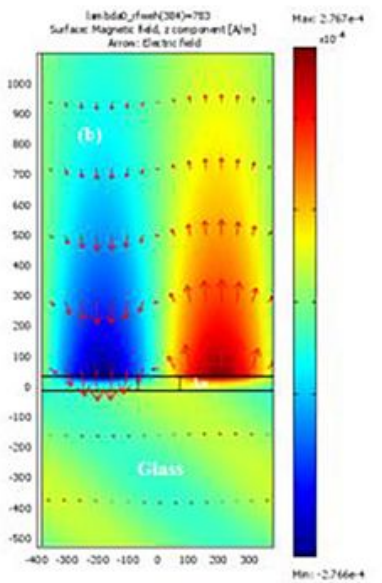

(f) $n=1.004, \lambda=839$

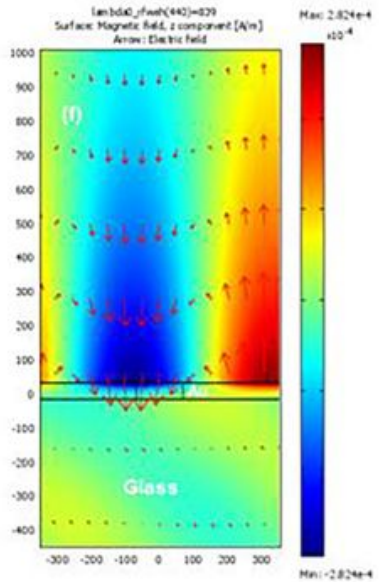

(j) $\mathrm{n}=1.008, \lambda=930$

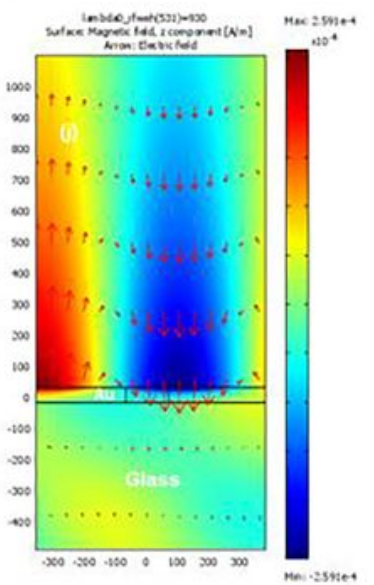

(c) $n=1.002, \lambda=808$

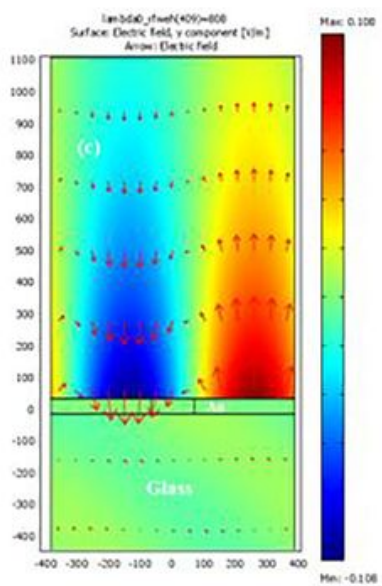

(g) $\mathrm{n}=1.006, \lambda=878$

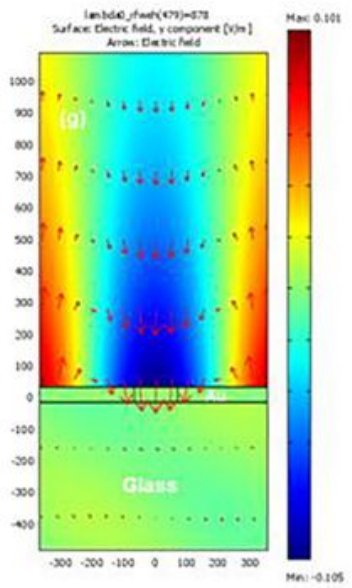

(k) $\mathrm{n}=1.01, \lambda=997$

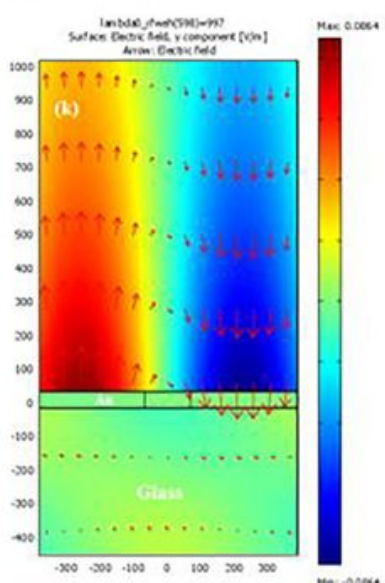

(d) $n=1.002, \lambda=808$

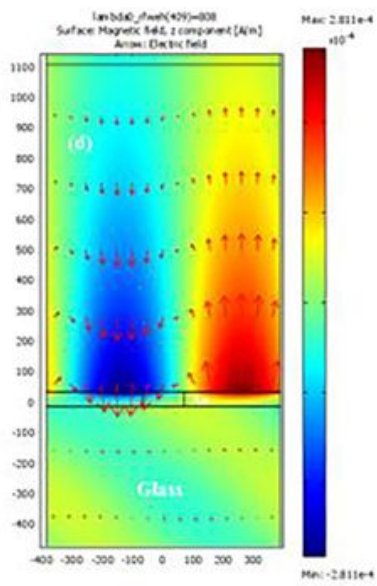

(h) $\mathrm{n}=1.006, \lambda=878$

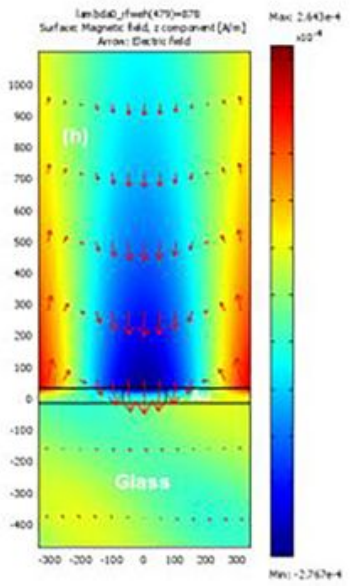

(I) $\mathrm{n}=1.01, \lambda=997$

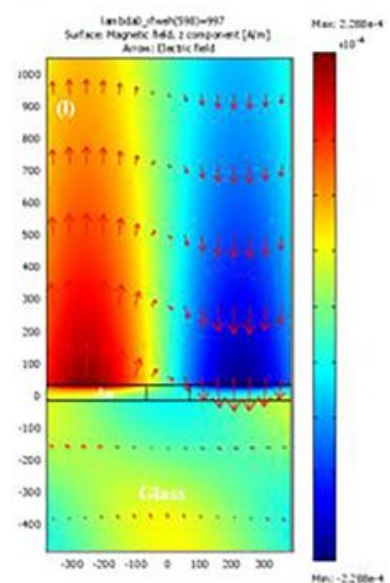

Figure 4

$\mathrm{y}$-component of electric field and z-component of magnetic field at incidence 430 with thickness $50 \mathrm{~nm}$. The y-components of electric field (Ey) are shown in fig.4 (a,c,e,g,i,k) and z-components of magnetic field are shown in Fig. 4 (b,d,f,h,j,l) 

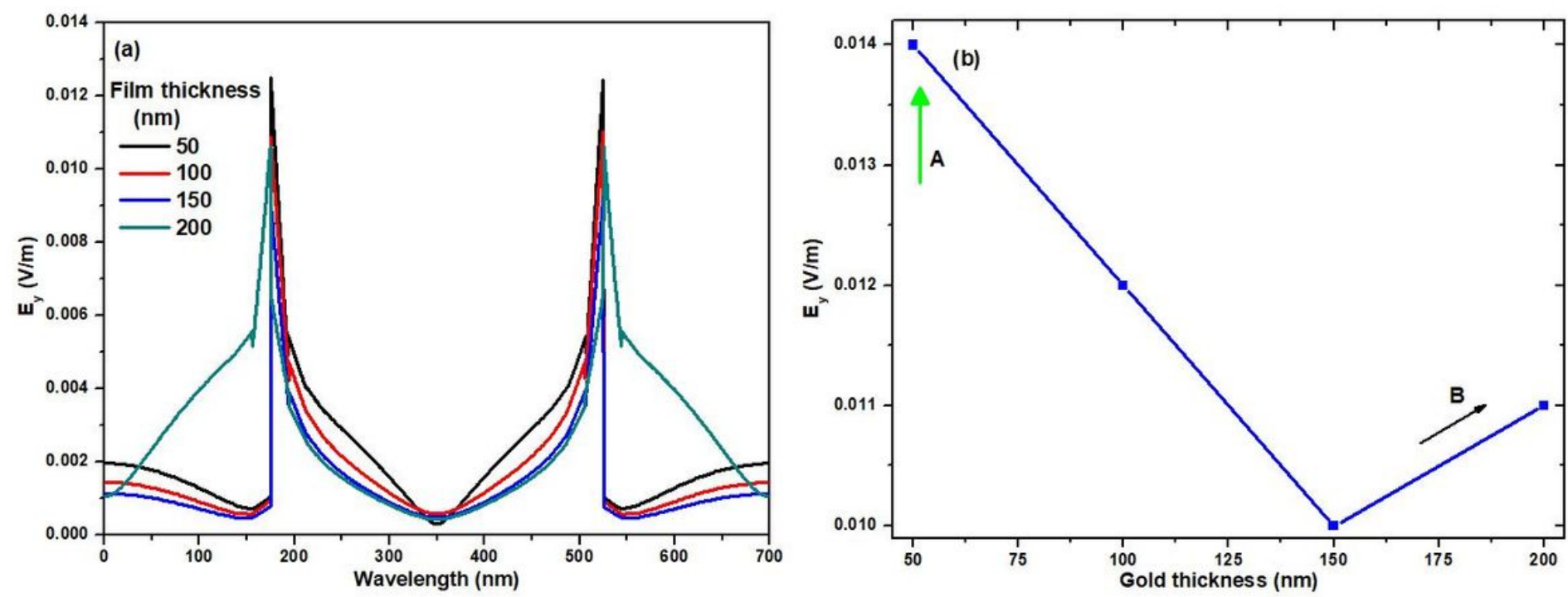

Figure 5

Cross sectional view of the electric field at different gold thicknesses. (a) y-component of electric field versus wavelength, (b) $y$-component of electric field versus gold thickness.

\section{Supplementary Files}

This is a list of supplementary files associated with this preprint. Click to download.

- graphicsabstract.jpg 